Published online 2017 April 13.

Abstract

\title{
Importance of Evaluation of Extra Cardiac Findings in Patients Undergoing MDCT Coronary Angiography
}

\author{
Mohammad Momeni, ${ }^{1,}{ }^{*}$ Maryam Farghadani, ${ }^{1}$ and Fatemeh Momeni ${ }^{1}$ \\ ${ }^{1}$ Isfahan University of Medical Sciences \\ "Corresponding author: Mohammad Momeni, Isfahan University of Medical Sciences. E-mail: dr.m.momeni@gmail.com
}

Received 2016 December 21; Accepted 2017 February 08.

\begin{abstract}
Background: Although the aim of cardiac computed tomographic angiography (CTA) is to visualize the cardiac, coronary, and great vessels, portions of noncardiac structures such as lung, mediastinum, bones and abdomen are visible on the scan. some have suggested that the scans should be evaluated in all field of view. Some other believe that reporting accidental findings can lead to unnecessary follow up and anxiety. prevalence and seriously of the findings can guided the need for reporting the accidental findings or not. The aim of our study was to evaluate the prevalence and seriously of accidental extracardiac findings on MDCT angiography of coronary arteries.

Methods: A total of 411 patients with suspected coronary artery disease were evaluated with MDCT during injection of contrast material. Images were reviewed for extracardiac findings which were classified as therapy needed, further work up needed, follow up needed and no further action needed.

Results: Extracardiac findings were detected in $24.33 \%$ patients. findings were classified as needed therapy (1.94\%), needed work up (8.02\%), needed follow up (10.94\%), no further action needed (3.40\%). Among the findings, which needed treatment, were lung infiltration and pulmonary emboli and one case of malignancy. hiatal hernia was the most common finding, followed by pulmonary nodules which were seen in $(2.9 \%)$ of patient.

Conclusions: Coronary artery MDCT can reveal important findings and disease in extracardiac structures. On other hand the prevalence of noncardiac findings on cardiac MDCT was significant. To avoid missing clinically important findings, all the field of cardiac MDCT should be evaluated carefully.
\end{abstract}

Keywords: Coronary CT Angiography, Extra-Cardiac Findings, Lung Diseases, Chest

This is an abstract presented in the 33rd Iranian congress of radiology (ICR) and the 15th congress of Iranian radiographic science association (IRSA) 